\title{
GOBERNANZA DIGITAL: DESARROLLO DE LAS TECNOPOLÍTICAS EN LOS PAÍSES DE AMÉRICA LATINA: UNA REVISIÓN SISTEMÁTICA DE LA LITERATURA
}

\section{DIGITAL GOVERNANCE: DEVELOPMENT OF POLITICAL TECHNO IN LATIN AMERICAN COUNTRIES: A SYSTEMATIC REVIEW OF LITERATURE}

\author{
Mónica Jimbo Santana Patricia Jimbo Santana²
}

\begin{abstract}
Resumen
La gobernanza digital es un tema nuevo dentro de la administración pública, que trata de resolver los problemas socioeconómicos, como la utilización de tecnologias digitales para apoyar los objetivos de políticas públicas y facilitar mejoras en el desarrollo de diferentes áreas especificas de gobierno, entre ellas salud, educación, seguridad y otras. Además, utiliza las nuevas tecnologías como las redes sociales, para comprometer a los ciudadanos en las decisiones. El objetivo es poner a disposición de la ciudadanía los datos del gobierno, de tal manera que las empresas, investigadores y ciudadanos puedan utilizar esos datos y desarrollar servicios y aplicaciones, de tal forma que se integre en un solo entorno al ciudadano con las instituciones públicas y privadas. En este artículo se realiza una investigación observacional y retrospectiva, sobre la base de una revisión sistemática de la literatura y mediante la búsqueda de artículos publicados desde el 2016 al 2020, en bases de datos como IEEE, Elservier, Springer, Oxford, Doaj, Scielo, Google Scholar. En los artículos analizados se hace referencia a seis grandes áreas, como son: los ciudadanos conectados, el control democrático de la ciudadanía, el sentir del ciudadano que es protagonista político, el activismo y las tecnologías, la información electoral, y las campañas electorales. Podemos concluir que no existem producciones cientificas relevantes en los paises de Latinoamérica; solo hay un artículo en ciertos paises, por lo que hay un nuevo segmento de investigación en este tema de gobernanza digital y tecnopolíticas.
\end{abstract}

Palabras claves

Gobernanza digital, tecnopolíticas, medios digitales, América Latina.

\begin{abstract}
Digital Governance is a new issue within the Public Administration, it seeks to solve socio-economic problems, such as using digital technologies to support public policy objectives, and facilitating improvements in the development of different specific areas of government, including health, education, security and other sectors; it uses new technologies such as social media, to engage citizens in decisions, the objective is to make government data available in such a way that companies, researchers and citizens can use that data and develop services and applications, integrate in a single environment the citizen with public and private institutions. This article conducts observational and retrospective research based on a systematic review of literature by searching for articles published from 2016 to 2020, in databases such as IEEE, Elservier, Springer, Oxford, Doaj, Scielo, Google Scholar. The articles analysed refer to six major areas, such as: connected citizens, democratic control of citizens, the feeling of the citizen who is a political player, activism and technologies, electoral information, and election campaigns. We can conclude that there are no relevant scientific productions in Latin American countries, there is only one article in certain countries. So, there is a new research segment on this issue of Digital Governance - Techno Policies.
\end{abstract}

\section{Keywords}

Digital governance, techno-policies, digital media, Latin America.

JEL: 021

$1 \quad$ Universidad Central del Ecuador, Quito - Ecuador (djimbo@uce.edu.ec).

2 Universidad Central del Ecuador, Quito - Ecuador (prjimbo@uce.edu.ec). 


\section{Introducción}

La sociedad ha incrementado la conectividad mundial en un $50 \%$, sea cual sea su renta, su latitud y longitud conectada a Internet. Se puede acceder a contenidos en segundos, lo que permite que aparezca con más intensidad el término de tecnopolíticas. Al hacer una referencia, podemos decir que actualmente la información que generamos en contenidos en un solo día, es la mismo que habíamos documentado, almacenado, archivado, en diez años, por lo tanto, vivimos en una sociedad conectada y altamente informada, que genera hoy mucho más contenidos de los que conocíamos hace diez años (Gutiérrez, 2019).

Cuando consideramos la velocidad de Internet del $4 \mathrm{G}$ al 5G, podemos decir que se va a multiplicar la capacidad de crear contenidos, dando lugar a una situación muy densa en términos de relación. Esto es importante para las organizaciones, para las personas, para los partidos políticos, para un Estado o para una ciudad, pues se va a multiplicar por dos la información que teníamos publicada o de cualquier tema que se esté operando (ONU 2020).

Si consideramos la Internet en Latinoamérica, que es la región que más crece, la digitalización se convierte en una pieza clave para el crecimiento (Katz, 2016), y representa un $13 \%$, y está por sobre la región sur del Asia y del Pacífico. En el 2010 los incrementos de movilidad, conectividad, los celulares y trasmisión de datos, especialmente desde la aparición del sms (short message service), han tenido un crecimiento exponencial y más con la utilización desde el 2007 de Facebook y Twitter, y actualmente TikTok (Palao P, 2020). En cualquier caso, la sociedad latinoamericana ha pasado directamente al $3 G$ y $4 G$ y eso va a seguir creciendo. En el último informe del Banco Mundial se hace referencia a que los ciudadanos que tienen un nivel de renta de uno a dos dólares al día, tienen un celular con conexión de datos (Gutiérrez A. 2019).

Este artículo está organizado así: la siguiente sección hace una breve revisión de la literatura sobre gobernanza digital, y las tecnopolíticas; posteriormente se presenta el método propuesto, se expone los resultados obtenidos $y$, finalmente, se resume las conclusiones y se propone algunas líneas de trabajos futuros.

\section{Revisión de la literatura}

\section{Gobernanza digital}

Es aplicar las nuevas tecnologías como redes sociales, para comprometer a los ciudadanos en las decisiones; permiten la disponibilidad de datos de gobierno para que las empresas, investigadores y ciudadanos puedan utilizar esos datos y desarrollar servicios y aplicaciones, integrar en un solo entorno al ciudadano con las instituciones públicas y privadas. Todo esto se conoce como las tecnopolíticas (Gutiérrez, 2016).

Se comenzó a implementar por etapas, lo primero era la incorporación de tecnología en el gobierno; el objetivo era desarrollar portales. Todos los gobiernos comenzaron a tener presencia en línea, automatizar los procesos administrativos y tratar de proveer acceso a los servicios, a través de sitios web.

Cuando se comenzó a desarrollar estos procesos, surgieron limitaciones porque la tecnología sola no podía entregar valor público y no podía entregar resultados concretos si no estaba asociada con cambios en las organizaciones, lo cual no es solamente automatizar y colocar las cosas en línea. Se desea mejores beneficios, se induce al cambio en los procesos de negocios y en las estructuras de gobierno, para entregar mejores beneficios (Reig R. Barneto 2019).

También se conoció que si se quería hacer todo en línea, no era solo la automatización y se vio que el desarrollo tecnológico 
por sí solo no entregaba valor público, que es necesario, más sitios webs de gobierno y sitios web municipales.

La segunda etapa fue reorganizar los procesos administrativos y la tecnología, con lo que había muchas bases de datos y procesos duplicados y hacer reingeniería para facilitar los recursos.

En la tercera etapa se ofreció servicio a las agencias con colaboración, lo que no había sido considerada como una cultura de gobierno.

Otra etapa fue ofrecer servicios entre las agencias, todo en colaboración y conectado, que estuviese de acuerdo con las necesidades de los ciudadanos, pero se vio que todo esto tampoco generaba valor público en sí, que se necesitaban otras cosas, y faltaba involucrar a la ciudadanía; conocer qué es lo que la ciudadanía quería entonces de gobierno digital. y se comenzó a hablar de gobernanza digital.

\section{Las tecnopolíticas}

En 1916, en un congreso, Otálora Cotrino Leonardo, en su publicación Una reflexión sobre el entorno o las devociones modernas, dijo "[...] creo que la política va a cambiarnos ante la economía si no cambian las relaciones sociales, pero como van a cambiar las relaciones sociales entonces cambiará la política".

Los proyectos de innovación o renovación políticas van siempre asociados a las estrategias o técnicas de tecnopolíticas de los años 2010-2011 en los Estados Unidos. Hoy vemos que la gente vive en dos lugares: en sus casas físicas y en sus casas digitales. Esta doble morada está convirtiéndose en la identidad de la gente. Hoy sabemos dónde la gente vive, qué piensa y qué siente y esas nuevas identidades están interactuando (Gutiérrez A. 2019).

Hoy las campañas políticas utilizan tecnología intensivamente y buscan la relación de estos dos mundos. Necesitamos saber qué piensa la gente, qué le preocupa y en donde está; estamos hablando de patrones abiertos, inteligencia artificial, que nos permite relacionar identidades líquidas con identidades físicas, que aportan mucha información socioeconómica (Vivanco. M 2019).

La tecnopolítica es la sociedad tecnificada que nos ha cambiado el escenario. Cuando pensábamos que teníamos todas las respuestas nos cambiaron las preguntas. La tecnopolítica es una irrupción contra la pereza intelectual. Si la conocemos suficientemente nos va a permitir ganar intelectualmente, ya que lucha contra la pereza y la obesidad intelectual. Las tecnopolíticas cambian las relaciones sociales en relaciones de poder.

Cuando estamos pensando en poder, nos enfocamos en un mundo de posición y tamaño. Asociamos tamaño y posición con poder y el poder de verdad es que estamos cambiando las cosas, modificando las ecuaciones, transformando la economía. Se está cambiando el pensamiento y la política tiene que ver con los conceptos ágiles, creativos, rápidos en comunidad y red.

Negocios pequeños, ágiles, conectados, frente a los lentos, grandes y analógicos; los segundos mueren porque no pueden competir con un negocio que está tecnificado.

En América Latina se tiene que ver las relaciones y contenidos, no todos relacionamos tamaño con posición sino relaciones y contenidos, entonces no se puede competir, están cambiando las ecuaciones de poder, las reglas de poder es una buena oportunidad para la política, por lo tanto, la inmaterialidad, lo creativo, las ideas, pueden hoy tener un papel de nuevo con capacidad competitiva frente a cualquiera.

En el libro Fin de poder del autor Moisés Naim, se explica por qué el poder se está transformando tan rápidamente; porque hay una multitud de agentes, con micropoderes y con capacidad de competir y con capacidad de crear alternativas, "por eso hoy es más fácil obtener poder por otros, más difícil 
de usar por el que lo tiene, y mucho más fácil de perder" (Naim, 2013).

La oferta política garantizada por las leyes, por nuestros sistemas y ordenamientos jurídicos, posesionales y políticos, protagonizados por partidos y sindicatos, que se ofrece a los ciudadanos cada cuatro o cinco años es decisiva; lo único que se quiere es el voto y luego adiós, una soberanía delegada (Angulo, 2020).

Los milenios tienen hoy en día la capacidad de fiscalizar, monitorear, opinar, contrastar, es decir tener voz. Se les ofrecía votar cada cuatro años y la gente tiene voces; este es el choque de la oferta política cada cuatro o cinco años, los milenios quieren voces diarias constantes y permanentes de manera activa. En el sistema nuestra arquitectura política es diferente y eso es lo que no se comprende y no se entiende bien, eso aumenta la desconfianza en Latinoamérica, una de las regiones más desconfiadas del mundo.

En América Latina está pasando lo que pasa en todas partes y pasa con mucha insistencia, es decir, si solo me ofreces ser decisivo cada cuatro o cinco años y mis voces no son escuchadas, más la corrupción, más el colapso de los servicios públicos, más las ineficiencias de las políticas públicas, aumenta la desconfianza, lo cual es evidente en las instituciones gubernamentales (Gutiérrez, 2016).

En un contexto deteriorado podemos decir que en estos últimos 10 años se ha aumentado la capacidad crítica, se ha incrementado la posibilidad de vigilar, fiscalizar y compartir, la gente tiene hoy una capacidad de intervención política que no es canalizada por la institucionalidad, y esto nos lleva lamentablemente a tener una gran brecha. Es el momento del ingreso de la tecnología, Facebook. Twitter y la insatisfacción con la democracia.

Cada vez más jóvenes conectados, cuando decimos los millennias (milenios), nos referimos a una generación que nació y creció con Google; para ellos la tecnología es un elemento inseparable de su vida cotidia- na; la generación conectada y relacionada (Gutiérrez, 2016).

Se dice que los milenios son los nacidos en el 2000, se ponen de moda en el 2013. Entre sus características: son egoístas, egocéntricos y vanidosos, mundo selfi, pero tienen sus méritos, son muy participativos, muy potentes, más todavía en países como el Ecuador con promedios de edad muy jóvenes. En una encuesta realizada en Ecuador, se indicó que los jóvenes son apolíticos, están aburridos con la política, con la oferta política, pero están muy concentrados en las causas sociales, voluntariado cívico, en propuestas políticas, que no sean marcados en casas políticas sino en causas sociales.

Esta generación no confía en un partido político, sino que lo considera como parte de un problema; es una generación políticamente independiente, indecisa, muy crítica y recelosa de la oferta actual. En este contexto, Internet pasa a ser un eco del sistema natural, por lo tanto, para esta ciudadanía, Internet no es un servicio de telecomunicaciones, es un derecho y lo viven como tal. Es como el derecho a participar de lo público, un bien común que hace que detrás de Internet existan infraestructuras, ordenadores, costes e inversiones.

Pero para esta generación, Internet es un derecho, no un servicio facturable. En este contexto aparece la tecnopolítica (Gutiérrez, 2016).

Existen seis grandes áreas donde se encuentra la tecnopolítica:

- Ciudadanos conectados.

- Control democrático de ciudadanía.

- Sentir ciudadano de participación y protagonismo político.

- El activismo y las tecnologías.

- Información electoral.

- Campañas electorales.

\section{a. Ciudadanos conectados}

Ciudadanos que se conectan por causas, por problemas, por convicciones, que su 
condición es móvil, que tienen capacidad autónoma para organizarse, que pueden participar, que amplifican sus propuestas y que entienden la influencia social como algo más importante que la decisión política; prefieren influir sobre el regulador, a la espera de que le toque el turno de votar, pero no van a exponerse al voto por ser influyentes. Por tanto, la tecnopolítica introduce un elemento donde la evaluación, de lo público y de lo político es permanente y donde la capacidad de intervención es continua (Angulo, 2020).

Es importante monitorear el trazo digital de las políticas públicas, así como el trazo digital de las campañas electorales, de partidos, de candidatos, de las instituciones. Esto hace que hoy tengamos una necesidad de observar y monitorear como nunca antes. Tenemos transparencia, contabilidades públicas, de vinculación de datos, que permite relaciones causales. Tenemos una capacidad extraordinaria de monitoreo. Es un cambio: antes no podíamos monitorear, debíamos esperar la rendición de cuentas y creer o no creer, y dar con la prensa libre y democrática como factor de fiscalización; ahora tenemos una fuerte capacidad tecnológica para monitorear.

\section{b. Control democrático de la ciudadanía}

Estamos hablando también de la capacidad de relacionar datos con causas y decisiones, y también de poder introducir estos elementos de chequeo y verificación en la agenda política mediática.

En las tecnopolíticas la Internet se ha convertido en un elemento, en un ecosistema que favorece la emancipación, la participación y la importancia de la voces en un entorno en donde las voces no eran suficientemente escuchadas; por ejemplo, más instituciones no contemplan el ejercicio democrático legitimado de sus funciones sin procesos adicionales de participación. Lo vemos en las políticas locales. Las decisiones van acompañadas por procesos de participación, con el objetivo, con el reforzamiento de la legitimidad (Humhries, 2020).

Hacer una política pública que transforme la viabilidad, la movilidad y el poner un tranvía por medio de una diagonal solo con votos individuales, no basta; se necesita de procesos para una nueva legitimación, por lo tanto, todas las administraciones están explorando capas adicionales de legitimidad a las políticas pública, y se están abriendo procesos de participación, donde lo tecnológico es el driver fundamental. Se quiere saber lo que se opina aunque, decido "yo", como regidor, alcalde o alcaldesa o grupo municipal o mayoría: yo decido votar por el candidato para que tome las decisiones. Se tiene un programa, se tiene mayoría en el consejo municipal, pero eso no es suficiente; se precisa un plus adicional que es escuchar con atención y también de legitimidad vía participación (Grandinetti et al., 2020).

Por lo tanto, las grandes movilizaciones y consultas muy abiertas van a ir creciendo en todas partes y la tecnología nos permite hacer estas cosas: deliberación, opinión, participación y también de voto democrático; por ejemplo, se está desarrollando en todo el mundo la tecnología para el voto masivo o al menos la consulta masiva a través de Internet, en donde no existe una urna, pero sí un elemento de revitalización para la democracia en términos de capacidad de escucha, y otras herramientas de colaboración.

\section{c. Sentir al ciudadano de participación $y$ protagonismo político}

La tecnopolítica hoy en día, se ve, se piensa y se comparte; se tiene que decir y se ha reducido la distancia entre pensar y hacer. Va con todo. La reducción entre pensar, decir, hacer, es extraordinaria y esto sí es un cambio, porque cuando pensábamos y decíamos algo necesitábamos un delegado sindical, ir a la puerta del partido y hacer una petición. Si no 
te escucharon se debe esperar unos cuatro años para tener el voto, y esto se terminó, pues el pensar, el decir, el hacer es una transición extraordinaria que hace sentir al ciudadano emancipación y protagonismo político. Por la tanto, el reporte, el denunciar, son los pilares fundamentales de la cultura democrática y por eso cada vez los teléfonos inteligentes se convierten en el instrumento básico del reporte ciudadano, gracias a la multitud de aplicaciones que pueden geolocalizar, que pueden compartir, que pueden asociar a la data, que pueden mapear, dando el reporte ciudadano (Labrador, 2020).

Existe un emprendimiento que se llama Axfordcity, que es una herramienta tecnológica de códigos abiertos, que se comparten en el mundo para tener la capacidad de mapear y hacer reportes en un gran espacio público. Esto no para de crecer. Las aplicaciones son las soluciones que se comparten, en términos de código, con otros entornos y con otras ciudades; el reporte es una gran herramienta (Gutiérrez, 2016).

\section{d. El activismo y las tecnologias}

Hoy en día pienso, digo, hago, denuncio, pero esto no es suficiente, pues ahora voy a pasar a la acción, no solo de denuncia, sino que convierto mi visión, mi denuncia, mi compromiso, mi participación en una acción y movilización. Cuando hablamos de tecno políticas estamos hablando de activismo, pues estamos descubriendo talentos naturales. Esta tecnología nos está haciendo creer y sentir que tenemos talentos y que podemos aflorarlos, que tenemos talento para mapear, hacer una fotografía, para escribir. Se ha dicho que no se podía, que no sabíamos y que teníamos que esperar y estamos descubriendo que sí se puede con la tecnología; esto es lo que está pasando. Todo tipo de activismo, en relación con la causa política, o sea el activismo político, es uno de los grandes elementos de la tecnopolítica (Castillo, 2020).

\section{e. Información electoral}

En un proceso electoral que deseamos conocer, para tener un voto formado e informado, cada vez más tenemos atajos informativos para saber cosas; es decir, aplicativos tecnológicos que se hacen y se descubren a través de cuestionarios sobre la ubicación dentro de un mapa electoral, bajo un sistema de metodología aplicado. Así, se consiguen los programas electorales de los partidos que nunca nadie lee y se los coloca en una base de datos comparada, se desarrolla preguntas para que la gente se autoreferencie con esas preguntas, en una escala de uno a seis grados (Barbera, 2020).

Esto es lo que está pasando en todas partes. También las grandes corporaciones están utilizando herramientas que tienen que ver con data, redes sociales y procesos electorales. Esto es la tecnopolítica.

\section{f. Campañas electorales}

En las campañas electorales lo principal es cómo conseguir el mover votos; esto también es tecno-política. Evidentemente, hoy tenemos una correlación que nos permite relacionar intereses con identidades, comportamientos con identidades. Hoy se puede conseguir saber en tiempo real las búsquedas, saber qué está buscando la gente en cualquier momento; cuáles son los intereses antes que saber las opiniones, porque puede ser que estos intereses acerquen más a la realidad que la opinión, ya que esta puede ser condicionada por varios factores; entonces se trabaja mejor y de forma asertiva con data, aplicando inteligencia artificial.

Por lo tanto, si tengo conversaciones, se las puede mapear semánticamente, se puede identificar vínculos, intereses, comunidades, relaciones, patrones, preferencias, tanto de intereses como de consumo. Es una relación directa entre los resultados y esto es comportamiento, no es opinión. (Estrada, 2020).

Para enfocarnos en las campañas electorales de hoy y del futuro y entender el com- 
portamiento, es muy importante referirnos a una gran capacidad de hacer, conocer e identificar, relacionar al elector con intereses y seguramente, con eso, tener la capacidad de segmentar mensajes, tantos como los que haga falta. Y esto es tecnopolítica (Sancho, 2020).

En estos procesos de tecnopolítica que estamos revisando, las campañas electorales, las políticas y las campañas van a quedar ergonómicamente representadas, ya no por los publicistas (eso se acabó); se necesita más, aunque algunos sean buenos publicistas se necesita data, análisis de comportamientos de los electores y quienes participan deben tener una democratización del talento para competir electoralmente, ya no con encuestas, pues esto hoy ya no es suficiente. Ahora hablamos de tecnopolítica.

\section{Materiales y métodos}

\section{Método}

Para la revisión sistemática de la literatura, se considera los lineamientos propuestos por Kitchenham (2009), para desarrollar la evolución de gobernanza digital y las tecnopolíticas en los países de América Latina. En la ejecución del protocolo se identificó la necesidad de revisión con una línea del tiempo desde el 2016, cuando comienzan las tecnopolíticas en gobernanza digital hasta el año 2020, y es cuando se especificaron las preguntas de investigación, se determinaron los términos de búsqueda y posteriormente se desarrolló y ejecutó el protocolo de revisión.

\section{Necesidad de la revisión}

La realización de esta revisión se hizo entre los años 2016-2019, aunque solo en el 2020 se presenta un gran número de publicaciones. En la tabla 1 se muestra los artículos sobre gobernanza digital y las tecnopolíticas, el país de referencia, así como el tema del artículo, mientras que en la tabla 2 se hace la misma referencia desde los años 2016 al 2019.

Tabla 1. Temas de gobernanza digital - tecnopolíticas en el 2020

\begin{tabular}{|c|c|l|l|l|}
\hline No. & $\begin{array}{c}\text { Año de } \\
\text { publicación }\end{array}$ & \multicolumn{1}{|c|}{ Temas } & $\begin{array}{c}\text { País de } \\
\text { referencia }\end{array}$ & Autores \\
\hline 1 & 2020 & $\begin{array}{l}\text { El Sistema de producción del Sistema de Pro- } \\
\text { ducción del Interior Bonaerense y las oportu- } \\
\text { nidades de inserción en torno a la bioecono- } \\
\text { mía y el paradigma tecnoeconómico. }\end{array}$ & Argentina & De Angelis lgnacio \\
\hline 2 & 2020 & $\begin{array}{l}\text { Porno-política y tecno-democracia en la } \\
\text { transición española, entre el destape y la } \\
\text { Constitución. }\end{array}$ & España & Germán Labrador Méndez \\
\hline 3 & 2020 & $\begin{array}{l}\text { Conflicto político, políticas públicas y ca- } \\
\text { Nacidades estatales: el caso del Registro } \\
\text { Agrarios de Argentina. }\end{array}$ & Argentina & Salgado, Rodrigo Martín \\
\hline 4 & 2020 & $\begin{array}{l}\text { REDD+policy translation and story lines in } \\
\text { Laos. }\end{array}$ & Estados Unidos & $\begin{array}{l}\text { S Ramcilovik-Suominen, } \\
\text { I Nathan }\end{array}$ \\
\hline 5 & 2020 & $\begin{array}{l}\text { Marco analítico de políticas digitales: el } \\
\text { caso de la estrategia nacional en México. }\end{array}$ & México & Beceerril - Velasco \\
\hline
\end{tabular}




\begin{tabular}{|c|c|c|c|c|}
\hline 6 & 2020 & $\begin{array}{l}\text { Políticas para promover la ciencia, tecnolo- } \\
\text { gía e innovación en la era digital. }\end{array}$ & Perú & $\begin{array}{l}\text { Fairlie Alan - Jessica } \\
\text { Portocarrero }\end{array}$ \\
\hline 7 & 2020 & $\begin{array}{l}\text { ¡Los barrios bajos nos quieren, los rebeldes } \\
\text { nos tendrán! ¡La tecnopolítica y la indigna- } \\
\text { ción, desde la Generación hasta la Rasca de } \\
\text { que la Troika está jodida! }\end{array}$ & Portugal & Sola Morales Salomé \\
\hline 8 & 2020 & $\begin{array}{l}\text { Error en el Sistema / Ctrl+Alt+Supr: Conec- } \\
\text { tar cuerpos y tecnologias desde perspecti- } \\
\text { vas transfeministas. }\end{array}$ & España & Martínez Pozo Lola \\
\hline 9 & 2020 & El trabajo en siglo XXI. & Estados Unidos & $\begin{array}{l}\text { Humhries Jane - Schnei- } \\
\text { der Benjamín }\end{array}$ \\
\hline 10 & 2020 & $\begin{array}{l}\text { Gubernamental neoliberal y transformación } \\
\text { excluyente en Argentina: el Prohuerta en el } \\
\text { lapso 2015-2019. }\end{array}$ & Argentina & García Oscar Ariel \\
\hline 11 & 2020 & $\begin{array}{l}\text { Políticas de promoción a la nanotecnología } \\
\text { en contexto semiperiférico: el caso de los } \\
\text { Fondos Argentinos Sectoriales. }\end{array}$ & Argentina & Surtayeva Sofya \\
\hline 12 & 2020 & $\begin{array}{l}\text { Ciberpolítica y Ciberdemocracia, Una ar- } \\
\text { queología del prefijo ciber. }\end{array}$ & Ecuador & Angulo Moncayo Natalia \\
\hline 13 & 2020 & $\begin{array}{l}\text { Evolución y estado actual de la ciencia y la } \\
\text { tecnología en República Dominicana. }\end{array}$ & $\begin{array}{l}\text { República } \\
\text { Dominicana }\end{array}$ & Riggio Giovanna - Olivares \\
\hline 14 & 2020 & $\begin{array}{l}\text { Apuntes teóricos sobre la reconfiguración } \\
\text { del espacio público y la emergencia de nue- } \\
\text { vas formas de acción política. }\end{array}$ & España & García Sánchez S. \\
\hline 15 & 2020 & $\begin{array}{l}\text { Observatorio de transparencia legislativa y } \\
\text { parlamento abierto. }\end{array}$ & España & Castillo LIV \\
\hline 16 & 2020 & $\begin{array}{l}\text { Comentario sobre el proyecto de Ley Ge- } \\
\text { neral en materia de ciencia tecnología e } \\
\text { innovación. }\end{array}$ & México & Espinoza Hernández \\
\hline 17 & 2020 & $\begin{array}{l}\text { La innovación educativa en América Latina: } \\
\text { lineamientos para la formulación de políti- } \\
\text { cas públicas. }\end{array}$ & Venezuela & $\begin{array}{l}\text { Ríos Cabrera Pablo, Ruiz } \\
\text { Bolívar Carlos }\end{array}$ \\
\hline 18 & 2020 & La muerte de la imaginación. & España & Jaume Andreu \\
\hline 19 & 2020 & $\begin{array}{l}\text { Sobre lo político y la política en jóvenes can- } \\
\text { didatos a diputaciones en elecciones presi- } \\
\text { denciales } 2018 .\end{array}$ & Argentina & Sancho RG \\
\hline 20 & 2020 & $\begin{array}{l}\text { Medios de comunicación y movilidad cogni- } \\
\text { tiva: series de políticas en streaming Pue- } \\
\text { bla México } 2018 \text {. }\end{array}$ & México & $\begin{array}{l}\text { Estrada Rodríguez - } \\
\text { Pérez K }\end{array}$ \\
\hline 21 & 2020 & $\begin{array}{l}\text { Internet y política: consecuencias políticas } \\
\text { y sociales de la revolución digital. }\end{array}$ & $\begin{array}{l}\text { Estados Unidos } \\
\text { - Ángeles }\end{array}$ & Barbera Pablo \\
\hline 22 & 2020 & $\begin{array}{l}\text { Tecnología, ragione ed experinza: intervista } \\
\text { as Andrew Feenberg. }\end{array}$ & Brasil & Mutchinick Joaquin \\
\hline 23 & 2020 & $\begin{array}{l}\text { As Fake News na era digital e a ausencia } \\
\text { de políticas públicas de educacao para uso } \\
\text { das TIC. }\end{array}$ & Brasil & $\begin{array}{l}\text { Parchen Charles Emma- } \\
\text { nuel- Almendra } \text { Freitas } \\
\text { Cinthia- Teixeira Cavalli } \\
\text { Tássia }\end{array}$ \\
\hline
\end{tabular}




\begin{tabular}{|c|c|l|l|l|}
\hline 24 & 2020 & $\begin{array}{l}\text { Tendencias y prácticas políticas de gobier- } \\
\text { no abierto a nivel municipal en Argentina. }\end{array}$ & Argentina & $\begin{array}{l}\text { Grandinetti Rita - Ezequiel } \\
\text { Miller }\end{array}$ \\
\hline 25 & 2020 & $\begin{array}{l}\text { Políticas nacionales de información científi- } \\
\text { ca y tecnología en México. }\end{array}$ & México & Guerrero Elda Mónica \\
\hline 26 & 2020 & $\begin{array}{l}\text { La argumentación como resolución de } \\
\text { problemas neocorporativistas de políticas } \\
\text { venezolanas. }\end{array}$ & Venezuela & Pineda Alicia \\
\hline
\end{tabular}

Tabla 2. Temas de gobernanza digital - tecno - políticas años 2016-2019

\begin{tabular}{|l|l|l|l|l|}
\hline No. & \multicolumn{1}{|c|}{$\begin{array}{c}\text { Año de } \\
\text { publicación }\end{array}$} & \multicolumn{1}{|c|}{ Temas } & \multicolumn{1}{c|}{$\begin{array}{c}\text { País de } \\
\text { referencia }\end{array}$} & \multicolumn{1}{c|}{ Autores } \\
\hline 1 & 2016 & $\begin{array}{l}\text { Una reflexión sobre el entorno a } \\
\text { las devociones modernas. }\end{array}$ & México & $\begin{array}{l}\text { Otálora Cotrino, } \\
\text { Leonardo }\end{array}$ \\
\hline 2 & 2016 & $\begin{array}{l}\text { Millennials en Latinoamérica: una } \\
\text { perspectiva desde el Ecuador. }\end{array}$ & Ecuador & Antoni Gutiérrez Rubí \\
\hline 3 & 2019 & $\begin{array}{l}\text { Política en red entre la cosmopoli- } \\
\text { tita y pseudopolítica. }\end{array}$ & Rusia & Putin A \\
\hline 4 & 2019 & Sociológica del Big data. & Argentina & Vivanco M \\
\hline 5 & 2019 & $\begin{array}{l}\text { Política e infroentrenamiento, re- } \\
\text { des sociales y discurso emocional. }\end{array}$ & España & $\begin{array}{l}\text { Reig R-M. Barneto - } \\
\text { A. Barradas }\end{array}$ \\
\hline
\end{tabular}

\section{Preguntas de investigación}

Las preguntas de investigación que se desarrollaron fueron:

- ¿Cómo ha evolucionado el estudio de la gobernanza digital?

- ¿Cómosehan desarrollado las tecnopolíticas en los países de Latinoamérica?

- ¿En qué países han sido desarrolladas las investigaciones?

- ¿Cuál es el tamaño de la muestra utilizada en las investigaciones?

\section{Criterios de inclusión}

Los criterios de elegir los artículos se fundamentaron en las siguientes características:

- Artículos escritos en idiomas: español, inglés y portugués.
- Publicaciones desde el 2016 al 2020.

- Artículos que incluyan las cadenas de búsqueda en el título; palabras claves o resumen.

- Artículos publicados en revistas o conferencias.

\section{Extracción}

Se seleccionaron varios términos principales y alternativos: gobernanza digital, tecnopolíticas, medios digitales, América Latina, con los que se estructuraron varias combinaciones de cadenas de búsquedas. En el 2020 el resultado son 49 artículos. Algunos artículos fueron descartados por no cumplir con uno o varios de los criterios de inclusión, esto se puede observar en la tabla 3. 
Tabla 3. Artículos encontrados, seleccionados e incluidos

\begin{tabular}{|l|c|c|}
\hline \multicolumn{1}{|c|}{ Repositorio } & $\begin{array}{c}\text { Artículos encontrados } \\
\text { en cadena de búsqueda }\end{array}$ & $\begin{array}{c}\text { Artículos incluidos } \\
\text { para la revisión }\end{array}$ \\
\hline Google Scholar & 24 & 16 \\
\hline Scielo & 8 & 4 \\
\hline Springer & 6 & 3 \\
\hline Oxford Journal & 4 & 3 \\
\hline Elserver & 4 & 2 \\
\hline IEEE & 3 & 3 \\
\hline Total & 49 & 31 \\
\hline
\end{tabular}

En total, de los artículos encontrados en la búsqueda, tenemos 49. Considerando las carac- terísticas de inclusión para revisión, quedaron 31 artículos, lo que se detalla en la figura 1.

Figura 1. Artículos encontrados en la cadena de búsqueda y los seleccionados para la revisión

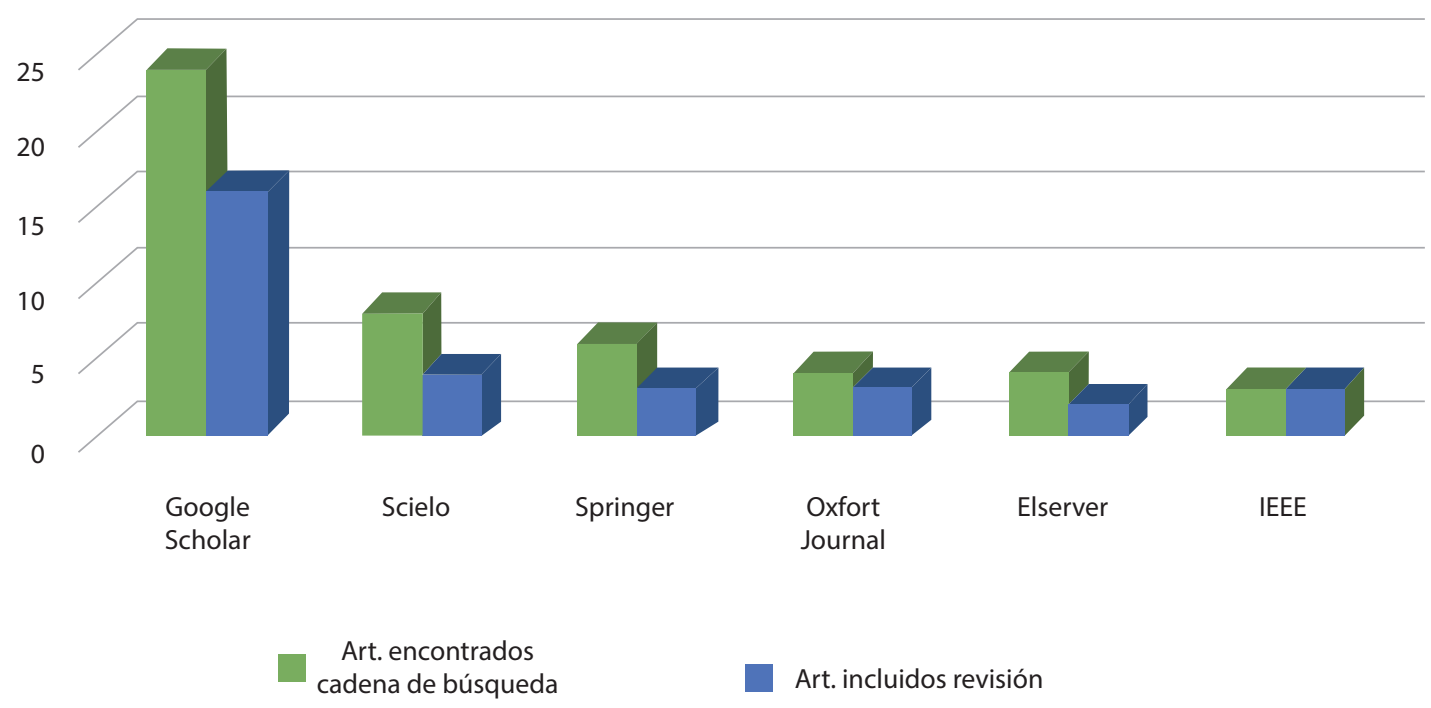

Se analizó en los artículos el contenido, la introducción, la metodología y conclusiones, los mismos que debían tener una estricta relación con las preguntas de investigación. En la tabla 3 se indican los resultados. Se elabora la matriz de extracción de datos con los siguientes campos: Id Artículo, año, título, autores y país de publicación.

De los 49 artículos se obtuvieron 31 que representan el $63.27 \%$, que cumplen con los criterios de inclusión.

\section{Analisis y resultados}

De los 31 artículos considerados, se efectuó el análisis para responder a las preguntas planteadas en la investigación.

¿Cómo ha evolucionado la gobernanza digital en los paises de América Latina?

En la tabla 4 se indica los artículos por años de publicación, y en la figura 3, el número de artículos incluidos para la revisión. 
Tabla 4. Artículos por años de publicación

\begin{tabular}{|c|c|}
\hline Años & $\begin{array}{c}\text { Número de artículos } \\
\text { incluidos para la revisión }\end{array}$ \\
\hline 2016 & 2 \\
\hline 2019 & 3 \\
\hline 2020 & 26 \\
\hline Total & 31 \\
\hline
\end{tabular}

Tomando una línea de tiempo desde el 2016 hasta el año 2020, tenemos, que el año de más publicaciones fue el 2020 con un $84.3 \%$. Se destaca que en el años 2016 se publicó 6 \% y en el año 2019 se publicó el $10 \%$. Esto nos indica que, como es un tema nuevo, este año 2020 se ha publicado más, lo que se representa en la figura 2.

Figura 2. Artículos por año de publicación

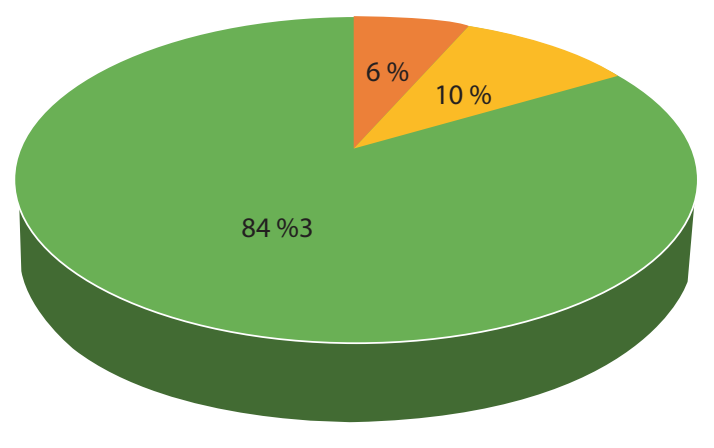

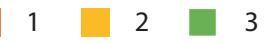

¿En quépaíses se ha publicado más y se han realizado más investigaciones sobre gobernanza digital y las tecnopoliticas?
En los países de Latinoamérica. Argentina es uno de los países que tiene más producción científica, seguido por México. En Europa tenemos a España. Este resultado también se puede observar en tabla 5 y en la figura 3.

Tabla 5. Artículos por países

\begin{tabular}{|l|c|}
\hline \multicolumn{1}{|c|}{ Países } & \multicolumn{1}{|c|}{$\begin{array}{c}\text { Número de artículos } \\
\text { incluidos para la revisión }\end{array}$} \\
\hline Argentina & 7 \\
\hline Brasil & 2 \\
\hline Ecuador & 2 \\
\hline España & 6 \\
\hline Estados Unidos & 3 \\
\hline México & 5 \\
\hline Perú & 1 \\
\hline Portugal & 1 \\
\hline República Dominicana & 1 \\
\hline Rusia & 1 \\
\hline Venezuela & 2 \\
\hline Total & 31 \\
\hline
\end{tabular}


Figura 3. Artículos por países

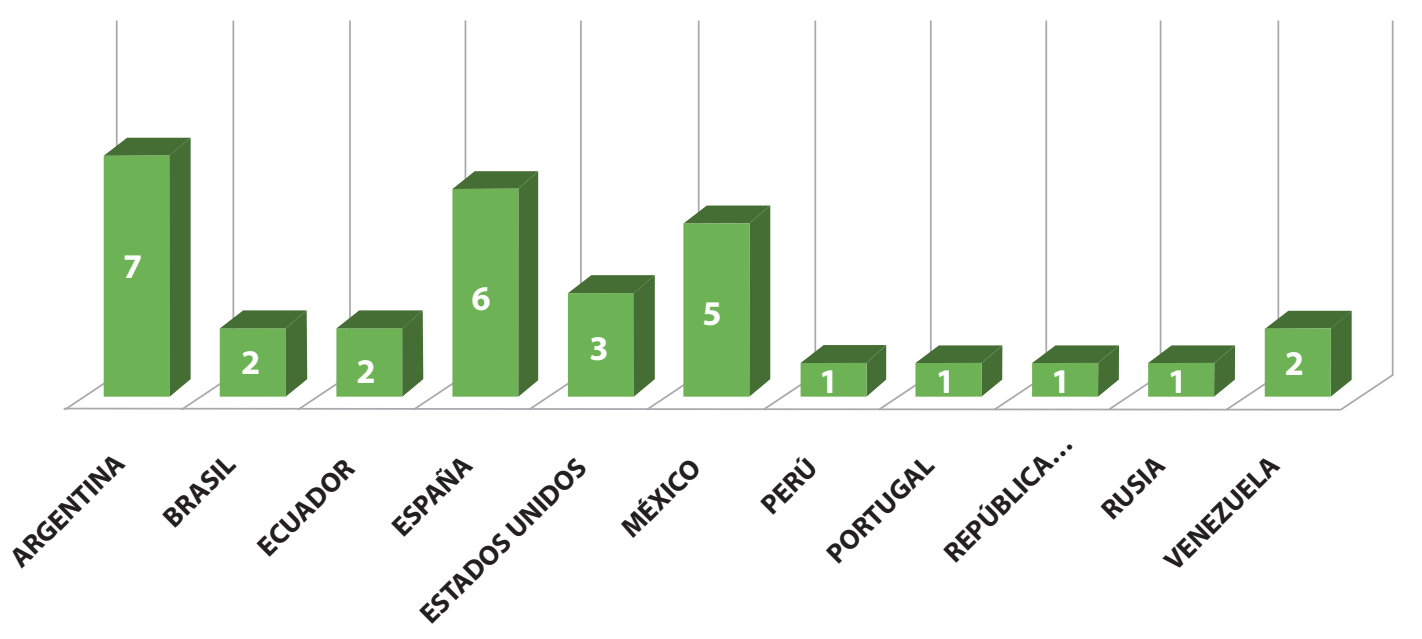

Primero realizamos un análisis por continente. Tenemos que en Europa se ha escrito acerca de gobierno digital y las tecnopolíticas en un $22.58 \%$. En todos los países de Latinoamérica se ha escrito el 74.29 $\%$ y el $3.13 \%$ corresponde a países de otros continentes. Esto es representativo ya que tenemos un impulso fuerte en la tecnología en América Latina.

El país que más artículos tiene es Argentina con un $22.58 \%$, seguido por México con el $16.13 \%$ y también existen publicaciones en Estados Unidos (9.68 \%) y Brasil (6.45\%). También observamos que en España es un tema de investigación con un porcentaje 19.35 $\%$, y existe un porcentaje bajo en la producción científica de otros países de Latinoamérica, como Venezuela, Perú, Ecuador, Portugal, Rusia y República Dominicana con el 25.81 \%.

¿Cuál es el tamaño de la muestra utilizada en la investigación?

Para el tamaño de la muestra se consideró el número de citas analizadas, lo que hace referencia al índice $\mathrm{H}$, el cual cuantifica tanto la productividad científica real, como el impacto científico; su autor fue Jorge Hirsch, su origen fue el 2005. La tabla 6 muestra el número de artículos seleccionados con el

número de citas determinadas por intervalos, evidenciando que se han tomado los que más citas tienen.

Tabla 6. Artículos por intervalo

\begin{tabular}{|l|c|}
\hline \multicolumn{1}{|c|}{ Intervalos } & $\begin{array}{c}\text { Número de artículos } \\
\text { por intervalo }\end{array}$ \\
\hline $1-5$ & 20 \\
\hline $6-10$ & 4 \\
\hline $11-15$ & 3 \\
\hline $16-20$ & 2 \\
\hline $21-25$ & 1 \\
\hline $25-$ más... & 1 \\
\hline Total & 31 \\
\hline
\end{tabular}

Entre los artículos científicos más citados, se encuentra un artículo de Ecuador del año 2016, tema: "Millennials en Latinoamérica: una perspectiva desde el Ecuador"; su auto,r Antoni Gutiérrez Rubí, tiene 18 citas; el siguiente más citado, tiene 16 citas, del autor Sola-Morales Salomé de Portugal, del año 2020, con el tema: "Los barrios bajos nos quieren, ¡los rebeldes nos tendrán! ¡La tecnopolítica y la indignación, desde la Generación hasta la Rasca de que la Troika está jodida!".

La mayoría de artículos está en el intervalo de 1 a 5 citas, existiendo 20 artículos. Otro 
de los más citados con 2 citas es "El trabajo en siglo XXI" de los autores Humhries Jane Schneider Benjaminy de Estados Unidos. Hay un artículo de Rusia de "Política en red entre la cosmopolítica y pseudopolítica" del autor Putin A. Otro de los citados es "Sociología del Big Data de Argentina". Otros autores: Grandinetti Rita - Ezequiel Miller con el artículo "Tendencias y prácticas políticas de gobierno abierto a nivel municipal en Argentina".

\section{Conclusiones y trabajos futuros}

Con las preguntas de investigación planteadas, la importancia de la revisión sistemática radica principalmente en determinar la existencia de estudios en el campo, con el tema de gobernanza digital y las tecnopolíticas, para extraer y analizar los diferentes procesos y técnicas de aplicación en los diferentes países del continente europeo como España y los países de América Latina, como han sido concebidos, diseñados e implementados.

La evolución de los artículos científicos de gobernanza digital es creciente. En el 2020 y sobre tecnopolíticas hay un 83 \%; en 2019 el 10 \%, y en 2016 el 6 \%- Esto es representativo por el impulso fuerte en la tecnología en América Latina, debido a que, durante la pandemia, la mayoría de los autores han realizado varias publicaciones.

Esto nos demuestra que existe un interés en la comunidad sobre estos campos que se han ido incrementado en el continente europeo $(n=7)$, en Latino América $(n=23)$ y en otros países como Rusia y República Dominicana. Los países de Latinoamérica que tienen los niveles más bajos, son: Perú, Ecuador y Venezuela $(n=2)$. En general, el gobierno digital y las tecnopolíticas parten de una filosofía política del software libre y los principios de la democracia. En los diferentes artículos se considera varias áreas como son las compras públicas, la participación ciudadana, la transparencia, la colabora- ción. Se realiza el análisis de casos en diferentes países como México, Chile, Colombia, así como una proyección hacia los años 2030 con el caso Costa Rica, la transparencia como objeto de desarrollo sostenible, y el área de la educación en gobiernos abiertos, con un proyecto.

Existe una gran batalla cultural de conceptos. Los ciudadanos tienen tanta tecnología en sus manos, que va a permitir que tengan mayor capacidad decisiva en lo político y en lo público. Hay capacidad de cálculo, transmisión, almacenamiento, conectividad. Se va a pasar del $4 G$ al $5 G$, entre otros saltos tecnológicos.

Por lo tanto, se va hacia un entorno, en donde, usadas responsablemente, estas tecnologías tendrán la oportunidad de aumentar la capacidad de la democracia. Sin embargo, existen muchos riesgos por parte de la gente que no quiere cambios, como por ejemplo, los grandes operadores de plataformas que no quieren perder su control, pero también se tiene la capacidad para detectarlo.

La Internet, las redes sociales, Facebook, Twitter, Tiktok, comercio electrónico, Big data, inteligencia artificial, generan un empoderamiento de la tecnología, del ciudadano común con la forma de hacer política. Esto habla de las tecnopolíticas inmersas dentro de la gobernanza digital.

Para trabajos futuros podemos analizar detenidamente los aplicativos como instrumentos digitales, las herramientas tecnológicas que se pueden aplicar y el análisis de la data para conocer costumbres y no opiniones, de esta forma se tiene una mayor presencia en la toma de decisiones políticas, dado que hoy el ciudadano quiere, solicita, exige una participación activa.

\section{Referencias bibliográficas}

Angelis, I. (enero de 2020). El sistema de producción del interior bonaerense y las oportunidades de inserción en torno a la bioecono- 
mía y el nuevo paradigma tecno económico. Observatorio de la Economía Latinoamericana. Revista Observatorio de la Economía Latinoamericana, (enero 2020). Disponible en: https://www.eumed.net/rev/ oel/2020/01/oportunidades-insercionbioeconomia.html//hdl.handle.net/ 20.500.11763/oel2001oportunidades-insercion-bioeconomia.

Castillo, L. J. V. (2020). Observatorio de transparencia legislativa y parlamento abierto. Estudios en derecho a la información, (10), 137-141.

Espinoza. R.. Comentario sobre el proyecto de Ley General en materia de Ciencia, Tecnología e Innovación. Revista de la Facultad de Derecho de México, Vol 70 (276-2), 757768.

Estrada Rodríguez, J. L. y Pérez Pérez, K. (2020). Medios de comunicación y movilidad cognitiva: series políticas en streaming, Puebla, México 2018. Anagramas-Rumbos y sentidos de la comunicación, 18(36), pp. 21-42.

Fairlie, A. y Portocarrero, J. (2020). Políticas para promover la ciencia, tecnología e innovación en la era digital. UDA AKADEM, Num (5), $61-81$

García S. (2020). Apuntes teóricos sobre la reconfiguración del espacio público y la emergencia de nuevas formas de acción política. Congreso internacional Move.net sobre movimientos sociales y TIC. Ponencia ISBN 978-84-09-20916-3

García, A. (2020). Gubernamentalidad neoliberal y transformación excluyente en Argentina: el ProHuerta en el lapso 2015-2019. Huellas, 24(1), 93-112

Grandinetti, R. y Miller, E. (2020). Tendencias y prácticas: Políticas de gobierno abierto a nivel municipal en Argentina. Revista iberoamericana de estudios municipales, (21), 89-112

Guerrero, E. M. (2020). Políticas nacionales de información científica y tecnológica en México. Revista española de documentación científica, 14(1), 34-46.

Gutiérrez, A. (2016). Millennials en Latinoamérica. Una perspectiva desde Ecuador. Po- nencia, Flacso, 11 Disponible en https:// www.gutierrez-rubi.es/2016/12/09/millennials-en-latinoamerica-una-perspectiva-desde-ecuador/

Katz, R. (2016) La digitalización: una clave para el futuro crecimiento de la productividad en América Latina, Centro de estudios de Telecomunicaciones de América Latina, Marka Magazine, Tecnologías Ideas e Innovación, Disponible en https://markamagazine.com/digitalizacion-clave-parael-crecimiento-de-la-productividad-enamerica-latina/

Kitchenham (2009) Systematic literature reviews in software engineering - A systematic literature review, Elservier p. 7-15.

Labrador Méndez, G. (2020). Una urna puede ser el mejor preservativo. Porno-política y tecno-democracia en la transición española, entre el destape y la Constitución. Mélanges de la Casa de Velázquez. Nouvelle série, (50-1), 85-114.

Moncayo, N. A. (2020). Ciberpolítica y ciberdemocracia. Una arqueología del prefijo ciber. URU-Revista de Comunicación y Cultura, (3), 17-29.

Morales, S. S. (2020). La tecnología y la generación rasca, 2(13), 30-48.

Mutchinick, J. (2020). Tecnologia, ragione ed esperienza: intervista ad Andrew Feenberg. Mechane, 181-188.

Otálora C. (2020). Una reflexión sobre el entorno las devociones modernas. Anales de Antropologìa 50, 148-151 Disponible en: https://www.bing.com/search?q=Una+re flexión+sobre+el+entorno+las+devocion es+modernas. \&cvid=4e9cf2cb898e4ee4 a7bd5e4b216f09cb\&aqs=edge..69i57.241 j0j4\&FORM=ANAB01\&PC = U531

ONU. (2020). Objetivos de desarrollo sostenible, 17 objetivos para transformar nuestro mundo, Infobae 3-07 Disponible en: https://www.infobae.com/opinion/2018/ 07/03/17-objetivos-para-transformarnuestro-mundo/.

Palao, P. (2020). El futuro crecimiento de las redes sociales: Instagram, Facebook y TikTok, Universidad de Alicante. Departamento de Comunicación y Psicología So- 
cia, Disponible en: http://rua.ua.es/dspace/handle/10045/107734

Parchen, C. E., de Almendra Freitas, C. O. \& de FBE Cavalli, T. T. (2020). As fake news na era digital e a ausência de políticas públicas de educação para o uso das tic's/fake news in digital age and the absense of public policies of education for the use of ict's. Revista Culturas Jurídicas, 7(16).

Pineda, A. (2020). La argumentación como resolución de problemas en el discurso neocorporativista de políticas venezolanas (1989-1994). Análisis crítico. Telos, 22(2), 445-461.

Pozo, L. M. (2020). Error en el sistema//Ctrl+ Alt+ Supr. Athenea digital, 20(1).

Putin, A. (2019). Política en red: entre la cosmopolítica y la pseudopolítica. Espacio, tiempo y forma, UNED Espacio, tiempo y forma Num 31, Disponible en: https://scholar. google.es/scholar?hl=es\&as_sdt $=0 \% 2 \mathrm{C5}$ $\& q=$ Putin\%2C+A.+(2019). +POLITICA+EN+ RED\%3A+ENTRE+LA+COSMOPOLITICA+Y $+\mathrm{LA}+$ PSEUDOPOLÍTICA.+Espacio\%2C+Tie $\mathrm{mpo}+\mathrm{y}+$ Forma. + Serie $+\mathrm{V} \% 2 \mathrm{C}+$ Historia $+\mathrm{C}$ ontemporá

Ramcilovik-Suominen, S. \& Nathan, I. (2020). REDD+ Policy Translation and Storylines in Laos, Journal of Political Ecology, 27(1). 436-455
Reig, R., Barneto, M. y Barradas, A. (2019). Política e infoentretenimiento: redes sociales y discurso emocional. La comunicación en el escenario digital. Actualidad, retos y prospectivas, La comunicación en el escenario digital. Actualidad, retos y prospectivas 735-783.

Riggio-Olivares, G. (2020). Evolución y estado actual de la ciencia y la tecnología en República Dominicana. Ciencia y Sociedad, 45(2), 7-32.

Ríos-Cabrera, P. y Ruiz-Bolívar, C. (2020). La innovación educativa en América Latina: lineamientos para la formulación de políticas públicas. Innovaciones Educativas, 22(32), 199-212.

Sancho, R. G. (2020). Sobre lo político y la política en jóvenes candidatos a diputaciones en elecciones presidenciales 2018. Revista Rupturas, 101-117.

Surtayeva, S. (2020). Políticas de promoción a la nanotecnología en contexto semiperiférico: el caso de los Fondos Argentinos Sectoriales. Ciencia, docencia y tecnología, 31(60 may-oct), 34-70.

Vivanco, M. (2019). Sociología del Big Data (Bachelor's thesis, Universidad Nacional de La Plata. Facultad de Humanidades y Ciencias de la Educación), Memoria Académica (UNLP-FAHCE), 05, 64-72 